\title{
Interfaces and Defects in Hybrid Molecular Beam Epitaxy Grown $\mathrm{NdTiO}_{3} / \mathrm{SrTiO}_{3}$ Heterostructures
}

\author{
Jong Seok Jeong ${ }^{1}$, Michael L. Odlyzko ${ }^{1}$, Peng Xu ${ }^{1}$, Bharat Jalan ${ }^{1}$ and K. Andre Mkhoyan ${ }^{1}$ \\ ${ }^{1}$ Department of Chemical Engineering and Materials Science, University of Minnesota, \\ Minneapolis, MN 55455, U.S.A.
}

Complex oxides with perovskite structure exhibit abundant electronic, spin, and structural phenomena including superconductivity, multiferroic behaviors, and Mott-Hubbard metal-insulator transition characteristics. The extreme sensitivity of these phenomena to crystal structure, bond length, bond angle, doping, and strain makes this material class very interesting for both fundamental physics and new technological applications [1-4]. Recent advances in thin film growth approach have made routine exercise to grow these materials in thin films/heterostructures forms with single-atomic-layer precision. This has led to the growth of a new class of system where layers consisting of single-atomic planes may enable the development of a new generation of electronic devices [5-7]. However, it should be noted that while rapid progress with these perovskite oxide thin films and heterostructures including demonstration of strain-stabilized non-equilibrium electronic properties, two-dimensional electron gases, quantum oscillations, and exotic magnetism and superconductivity has been made, the origin of many of these phenomena are not yet fully understood.

Here, we focus on investigating interfaces and defects in $\mathrm{NdTiO}_{3} / \mathrm{SrTiO}_{3}$ (NTO/STO) heterostructures using analytical scanning transmission electron microscopy (STEM). Composition, atomic or crystal structure and electronic properties were analyzed by the STEM with high-angle annular dark-field (HAADF) imaging using an aberration-corrected monochromatic FEI Titan G2 60-300 equipped with a Gatan Enfinium EELS spectrometer and a Super-X EDX system.

Pure-phase, NTO films were epitaxially grown on (001) (La0.3Sr0.7)(A10.65Ta0.35)O3 (LSAT) substrates using a hybrid molecular beam epitaxy approach [8,9]. For NTO/STO superlattice structures, stoichiometric STO $(3 \mathrm{~nm})$ and NTO $(6 \mathrm{~nm})$ thin layers were alternately grown on the substrate (Figure 1). NTO films have clear interfaces and uniform thickness over the entire specimen (Figure 1a). HAADF-STEM images show that the bottom interfaces of NTO films (the green lines in Figure 1b) are abrupt while their top interfaces (the blue lines in Figure 1b) are somewhat broadened in the range of 1-3 atomic layers (see the intensity profile in Figure 1b). In addition to the interface broadness, interestingly, NTO films grown under specific growth conditions display defects: one localized over a unit cell and another propagating through films (Figure 2). STEM results show that even though there are the propagating defects, the bottom interface is still atomically sharp, which reveals that the interface broadness is influenced by not only growth conditions but interface property itself.

The localized defect is explained by $45^{\circ}$ rotation of a NTO unit cell on the electron beam direction as shown in the EDX maps (Figure 2b). They are formed due to compressive strain in NTO films resulting from the larger unit cell of NTO. We will present EELS and EDX study for the interfaces and defects in detail, and the relationship between the broadness of the interfaces with their polarity will be discussed $[10,11]$.

References: 
[1] J. Biscaras et al., Nat. Commun. 1 (2010), p. 89.

[2] A. D. Caviglia et al., Phys. Rev. Lett. 105 (2010), p. 236802.

[3] Y. Kozuka et al., Nature 462 (2009), p. 487.

[4] A. Brinkman et al., Nature Mater. 6 (2007), p. 493.

[5] A. Ohtomo et al., Nature 419 (2002), p. 378.

[6] W. Tian et al, Appl. Phys. Lett. 89 (2006), p. 092905.

[7] A. Yoshikawa et al., Appl. Phys. Lett. 90 (2007), p. 073101.

[8] L. King et al., Appl. Phys. Lett. 59 (1991), p. 3045.

[9] K. Endo et al., Thin Solid Films 206 (1991), p. 143.

[10] N. Nakagawa et al., Nat. Mater. 5 (2006), p. 204.

[1 1 ] This work was supported in part by C-SPIN, one of the six centers of STARnet, a Semiconductor Research Corporation program, sponsored by MARCO and DARPA.
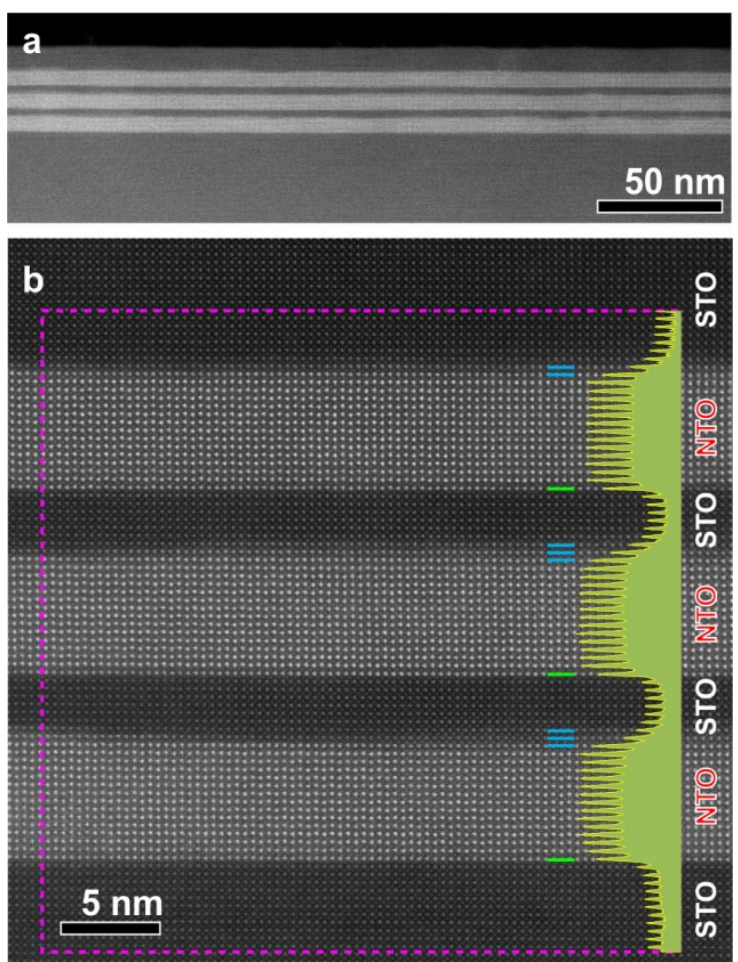

Figure 1. (a) Low-magnification and (b) high-resolution HAADF-STEM images of NTO/STO heterostructures.

A laterally-averaged intensity profile along the film depth direction in the boxed region was superimposed and interfaces were indicated by the green (bottom) and blue (top) lines.

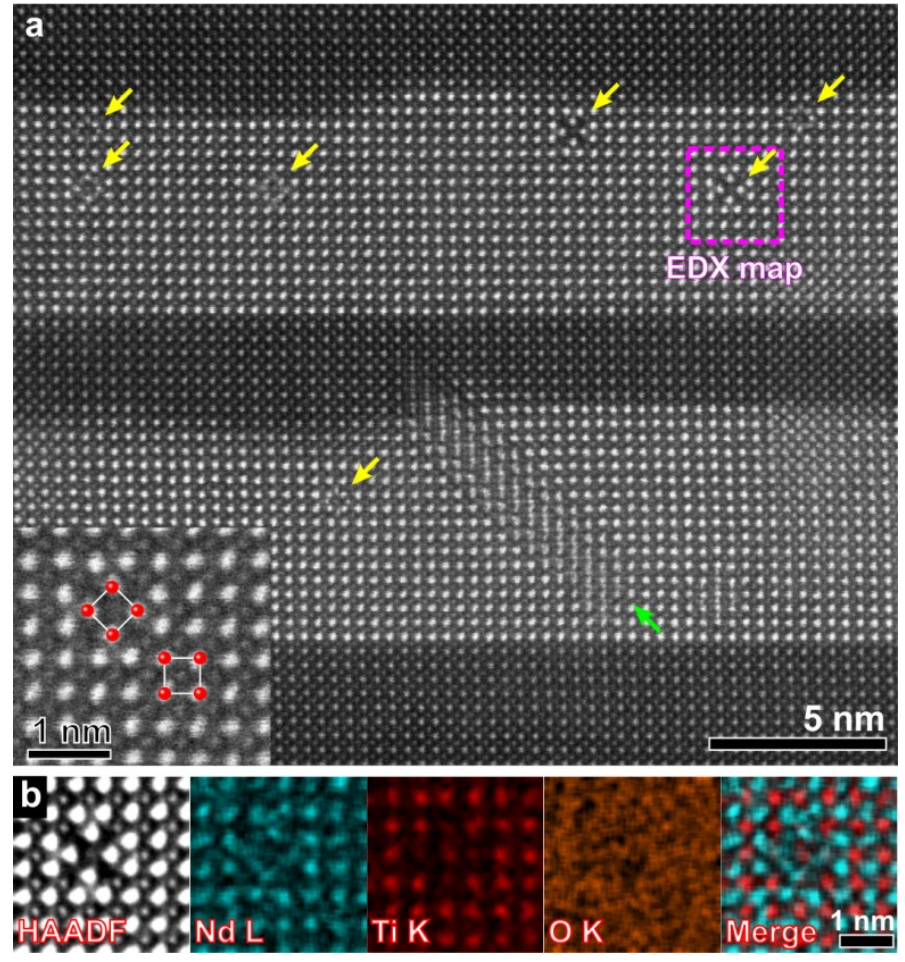

Figure 2. (a) High-resolution HAADF-STEM image showing two different defects: one localized (the yellow arrows) and one propagating (the green arrow). There are also two different interfaces (broad and sharp) in NTO/STO heterostructures. (b) EDX elemental maps in the localized defect showing $45^{\circ}$ rotation of a pseudo-cubic NTO unit (see the inset in a). 